\title{
A Necessary Condition for the Existence of a Certain Resolvable Pairwise Balanced Design
}

\author{
Satoru Kadowaki ${ }^{1, *}$, Sanpei Kageyama ${ }^{2}$ \\ ${ }^{1}$ Department of Science, National Institute of Technology, Matsue College, Matsue, Japan \\ ${ }^{2}$ Emeritus Professor of Hiroshima UniversityHiroshima University, Higashi-Hiroshima, Japan
}

\section{Email address:}

s.kadowaki@matsue-ct.jp (S. Kadowaki),pkyfc055@ybb.ne.jp (S. Kageyama)

*Corresponding author

\section{To cite this article:}

Satoru Kadowaki, Sanpei Kageyama. A Necessary Condition for the Existence of a Certain Resolvable Pairwise Balanced Design. International Journal of Theoretical and Applied Mathematics. Vol. 6, No. 2, 2020, pp. 28-30. doi: 10.11648/j.ijtam.20200602.12

Received: January 14, 2020; Accepted: February 19, 2020; Published: April 2, 2020

\begin{abstract}
A mathematical topic using the property of resolvability and affine resolvability was introduced in 1850 and the designs having such concept have been statistically discussed since 1939. Their combinatorial structure on existence has been discussed richly since 1942 . This concept was generalized to $\alpha$-resolvability and affine $\alpha$-resolvability in 1963 . When $\alpha=1$, they are simply called a resolvable or an affine resolvable design, respectively. In literature these combinatorial arguments are mostly done for a class of block designs with property of balanced incomplete block (BIB) designs and $\alpha$-resolvability. Due to these backgrounds, Kadowaki and Kageyama have tried to clarify the existence of affine $\alpha$-resolvable partially balanced incomplete block (PBIB) designs having association schemes of two associate classes. The known 2-associate PBIB designs have been mainly classified into the following types depending on association schemes, i.e., group divisible (GD), triangular, Latin-square $\left(\mathrm{L}_{2}\right)$, cyclic. First, it could be proved that an affine $\alpha$-resolvable cyclic 2 -associate PBIB design does not exist for any $\alpha \geq 1$. Also, Kageyama proved the non-existence of an affine $\alpha$-resolvable triangular design for $1 \leq \alpha \leq 10$ in 2008 . Furthermore, the existence of affine resolvable GD designs and affine resolvable $\mathrm{L}_{2}$ designs with parameters $v \leq 100$ and $r, k \leq 20$ was mostly clarified by Kadowaki and Kageyama in 2009 and 2012. As a result, only three designs (i.e., two semi-regular GD designs, only one $\mathrm{L}_{2}$ design) are left unknown on existence within the practical range of parameters. In the present paper, a necessary condition for the existence of a certain resolvable pairwise balanced (PB) design (i.e., some block sizes are not equal) is newly provided. Existence problems on PB designs are far from the complete solution. By use of the necessary condition derived here, we can also show a non-existence result of the affine resolvable $\mathrm{L}_{2}$ design which is left as only one unknown among $\mathrm{L}_{2}$ designs.
\end{abstract}

Keywords: Affine Resolvability, Resolvability, PB Design, $\mathrm{L}_{2}$ Design

\section{Introduction}

A PB design with parameters $v, b, r, K, \lambda$ is a collection of $b$ subsets (blocks) of a set of $v$ treatments such that the size of each block is an element of a set $K$, each treatment occurs in exactly $r$ different blocks and any two treatments occur in exactly $\lambda$ blocks. Even now, existence problems on some PB designs are also discussed (see, $[1,3,5,9,10]$ ).

A $\mathrm{L}_{2}$ design with parameters $v=s^{2}, b, r, k, \lambda_{1}, \lambda_{2}$ is defined as an arrangement of $s^{2}$ treatments, divided into an $s \times s$ array, into $b$ blocks of size $k$ each such that any two treatments (called the 1st associates) in the same row or in the same column of the array occur in exactly $\lambda_{1}$ blocks, whereas any two treatments (called the 2nd associates)not in the same row and in the same column of the array occur in exactly $\lambda_{2}$ blocks. Here $s \geq 2$.

A block design is said to be resolvable if its $b$ blocks can be grouped into $r$ resolution sets of $b / r$ blocks each such that in each resolution set any treatment occurs exactly once. A resolvable block design is said to be affine resolvable if any two blocks belonging to different resolution sets intersect in the same number of treatments. The combinatorial properties for (affine) resolvable BIB designs or (affine) resolvable PBIB designs have been discussed in literature (see, $[2,4,6,7,8,11$, 
12]). Furthermore, the characterization and the existence for (affine resolvable) $\mathrm{L}_{2}$ designs have been discussed in literature (see, $[4,8,11]$ ).

Kadowaki and Kageyama [8] produced comprehensive combinatorial findings on affine resolvable PBIB designs including $\mathrm{L}_{2}$ designs. In this paper, a new necessary condition for the existence of a certain resolvable PB design is presented. By use of the necessary condition, a non-existence result of an affine resolvable $\mathrm{L}_{2}$ design is newly shown. This $\mathrm{L}_{2}$ design has been left as only one unknown case in Kadowaki and Kageyama [8].

\section{Statements}

At first, the following is easily shown by taking $s$ treatments of the first associates in the $\mathrm{L}_{2}$ design.

Lemma 1. The existence of a resolvable $\mathrm{L}_{2}$ design with parameters $v=s^{2}, b, r, k, \lambda_{1}, \lambda_{2}$ implies the existence of a resolvable PB design with parameters $v=s, b, r, K=$ $\left\{k_{j}\right\}, \lambda_{1}$, where $k_{j} \leq k$ for $j=1,2, \cdots, b$.

Next, the main result can be established by some combinatorial calculation as the following shows.

Theorem 1. For $v \geq 3$ and $b=2 r$, a necessary condition for the existence of a resolvable PB design with parameters $v, b, r, K, \lambda$ is that $r-\lambda$ is even.

Proof. Consider the incidence matrix $N$ of the resolvable PB design with parameters $v, b=2 r, r, K, \lambda$. Let

$$
N=\left[\begin{array}{c}
\boldsymbol{v}_{1} \\
\boldsymbol{v}_{2} \\
\vdots \\
\boldsymbol{v}_{v}
\end{array}\right]
$$

where $\boldsymbol{v}_{i}$ is the $i$-th row vector of $N$ for $i=1,2, \cdots, v$.

Since the PB design is resolvable and $b=2 r$, each of resolution sets consists of two columns. Hence, by permuting columns of $N$ properly, without loss of generality we can have the same incidence structure corresponding to the position from the first to $2 \lambda$ columns in the first two rows, $\boldsymbol{v}_{1}, \boldsymbol{v}_{2}$, of $N$. Thus, by letting $\boldsymbol{v}_{i}=\left(\boldsymbol{v}_{i 1}, \boldsymbol{v}_{i 2}\right)$ where $\boldsymbol{v}_{i 1}$ is of size $1 \times 2 \lambda$ and $\boldsymbol{v}_{i 2}$ is of size $1 \times(b-2 \lambda)$ in $N$, we have $\boldsymbol{v}_{11}=\boldsymbol{v}_{21}$. Here $2 \lambda \leq b-2$. That is, $\boldsymbol{v}_{11}{ }^{t} \boldsymbol{v}_{21}=\lambda$ and then $\boldsymbol{v}_{12}{ }^{t} \boldsymbol{v}_{22}=0$. Also, since $v \geq 3$, under the present set-up, it follows that for the third row $\boldsymbol{v}_{3}$ of $N$

$$
\boldsymbol{v}_{11}{ }^{t} \boldsymbol{v}_{31}=\boldsymbol{v}_{21}{ }^{t} \boldsymbol{v}_{31}\left(=\lambda_{3}, \text { say }\right) .
$$

Note that $\lambda_{3} \leq \lambda$.

Further let

$$
\boldsymbol{v}_{12}{ }^{t} \boldsymbol{v}_{32}=\lambda_{13} \text { and } \boldsymbol{v}_{22}{ }^{t} \boldsymbol{v}_{32}=\lambda_{23} .
$$

Then, since $\boldsymbol{v}_{12}{ }^{t} \boldsymbol{v}_{22}=0$, it holds that

$$
\lambda_{13}+\lambda_{23}=r-\lambda .
$$

Furthermore, it follows that in the total PB design

$$
\lambda_{3}+\lambda_{13}=\lambda \text { and } \lambda_{3}+\lambda_{23}=\lambda
$$

which show $\lambda_{13}=\lambda_{23}$. This fact implies by the equation $\lambda_{13}+\lambda_{23}=r-\lambda$ that $r-\lambda$ is even. The proof is complete.

Now, by use of Lemma 1 and Theorem 1, we can easily obtain the following result.

Corollary 1 . For $s \geq 3$ and $b=2 r$, a necessary condition for the existence of a resolvable $\mathrm{L}_{2}$ design with parameters $v=s^{2}, b, r, k, \lambda_{1}, \lambda_{2}$ is that $r-\lambda_{1}$ is even.

Finally, the following result can be obtained.

Corollary 2. An affine resolvable $\mathrm{L}_{2}$ design with parameters $v=36, b=20, r=10, k=18, \lambda_{1}=7, \lambda_{2}=4$ does not exist.

Proof. If there exists the design with $v=6^{2}$, then, by considering 6 consecutive treatments of the first associates, a resolvable PB design with parameters $v=6, b=20, r=$ $10, K, \lambda=\lambda_{1}=7$ must be obtained. However, since $r-\lambda=3$ (odd), Corollary 2 shows the required result.

\section{Concluding Remark}

Since an affine resolvable design is also a resolvable design, by Corollary 3,the unknown status “?" for a design (stated in Corollary 3) of No. 17 in Table 3.6.2 of Kadowaki and Kageyama [8] should be revised as "Non-E" as a non-existence. Thus, we could completely clarify the existence status of affine resolvable $\mathrm{L}_{2}$ designs with admissible parameters within the scope of $v \leq 100$ and $r, k \leq 20$.

Incidentally, we further investigate affine resolvable $\mathrm{L}_{2}$ designs with admissible parameters $v, b, r, k, \lambda_{1}, \lambda_{2}$ within the scope of

$$
101 \leq v \leq 200 \text { and } 21 \leq r \text { or } k \leq 100 .
$$

Among them, there are more 9 possible designs with $b=2 r$, where 5 designs satisfy $r-\lambda_{1}=$ odd and then they are nonexistent (by Corollary 2). Other 4 designs have $r-\lambda_{1}=$ even. The 4 designs have the following admissible parameters and existence status, where "Comment" shows the existence of such designs without affine resolvability and quoted Theorems come from Raghavarao [11], while ? denotes unknown status of corresponding designs.

Thus, only one design of No. 2 is unknown as an affine resolvable $\mathrm{L}_{2}$ design with parameters $v, b=2 r, r, k, \lambda_{1}, \lambda_{2}$ within the scope of

$$
v \leq 200 \text { and } r \text { or } k \leq 100
$$


Table 1. Possible affine resolvable $L_{2}$ designs with $b=2 r$.

\begin{tabular}{llllllllll}
\hline No & $v$ & $b$ & $r$ & $k$ & $\lambda_{1}$ & $\lambda_{2}$ & $q_{2}$ & Affine resolvability & Comment \\
\hline 1 & 64 & 28 & 14 & 32 & 10 & 6 & 16 & Non-E (Theorem 12.6.6) & Theorem 8.10.2 \\
2 & 64 & 98 & 49 & 32 & 21 & 25 & 16 & $?$ & $?$ \\
3 & 144 & 44 & 22 & 72 & 16 & 10 & 36 & Non-E (Theorem 12.6.6) & Theorem 8.10.2 \\
4 & 144 & 242 & 121 & 72 & 55 & 61 & 36 & Non-E (Theorem 12.6.5) & $?$ \\
\hline
\end{tabular}

\section{References}

[1] T. Beth, D. Jungnickel and H. Lenz, Design Theory, Volume 1, 2nd Edition, Cambridge Univ. Press, UK, 1999.

[2] R. C. Bose, A note on the resolvability of balanced incomplete block designs, Sankhyā A 6 (1942), 105-110.

[3] T. Caliński and S. Kageyama, Block Designs: A Randomization Approach, Vol. II: Design, Lecture Notes in Statistics 170, Springer, New York, 2003.

[4] W. H. Clatworthy, Tables of Two-Associate-Class Partially Balanced Designs, NBS Applied Mathematics Series 63, U.S. Department of Commerce, National Bureau of Standards, Washington, D.C., 1973.

[5] P. J. Dukes and E. R. Lamken, Constructions and uses of incomplete pairwise balanced designs. Des. Codes Cryptogr. 12 (2019), 2729-2751.

[6] S. Furino, Y. Miao and J. Yin, Frames and Resolvable Designs, CRC Press, Boca Raton FL, 1996.
[7] S. Kadowaki and S. Kageyama, A 2-resolvable BIBD(10, 15, 6, 4, 2) does not exist, Bulletin of the ICA. 53 (2008), $87-98$.

[8] S. Kadowaki and S. Kageyama, Existence of affine $\alpha$-resolvable PBIB designs with some constructions, Hiroshima Math. J. 39 (2009), 293-326. Erratum, Hiroshima Math. J. 40 (2010), p. 271.

[9] E. Kramer and D. Kreher, $t$-Wise balanced designs. In: Handbook of Combinatorial Designs (ed. by C. J. Colbourn and J. H. Dinitz), Second edition, CRC Press, pp. 657-663.

[10] O. P. Popoola and B. A. Oyejola, Construction of congruent classes of pairwise balanced designs using lotto designs. Annals. Computer Science Series. 1 (2019), 127- 134.

[11] D. Raghavarao, Constructions and Combinatorial Problems in Design of Experiments, Dover, New York, 1988.

[12] S. S. Shrikhande and D. Raghavarao, Affine $\alpha$-resolvable incomplete block designs, Contributions to Statistics, Volume presented to Professor P. C. Mahalanobis on his 75th birthday, Pergamon Press, Oxford and Statistical Publishing Society, Calcutta, 1963, 471-480. 